\title{
Enxerto de Bypass de Artéria Coronária Guiado por Angiografia ou Fisiologia: Uma Metanálise
}

\author{
Physiology or Angiography-Guided Coronary Artery Bypass Grafting: A Meta-Analysis \\ José Martins, ${ }^{1}$ ำ Vera Afreixo, ${ }^{2}$ Luís Santos, ${ }^{1}$ Luís Fernandes, ${ }^{3}$ Ana Briosa ${ }^{1}$ \\ Baixo Vouga Hospital Centre, ${ }^{1}$ Aveiro - Portugal \\ CIDMA/IBIMED/Department of Mathematics, University of Aveiro, ${ }^{2}$ Aveiro - Portugal \\ Centre for Health Economics, University of York, ${ }^{3}$ York - Reino Unido
}

\section{Resumo}

Fundamento: Enquanto a angiografia coronária invasiva é considerada padrão outro para o diagnóstico da doença arterial coronariana (DAC), envolvendo os vasos coronários epicárdicos, a revascularização coronariana guiada por fisiologia representa uma prática padrão ouro contemporânea para a administração invasiva de pacientes com DAC intermediária. Porém, os resultados de longo prazo da avaliação da gravidade da estenose por meio da fisiologia, em comparação à angiografia como guia para a cirurgia de bypass - enxerto de bypass de artéria coronária (CABG), ainda são incertos. Esta metanálise visa avaliar os resultados clínicos de um CABG guiado por fisiologia em comparação a um CABG guiado pela angiografia.

Objetivos: Buscamos determinar se os resultados entre um CABG guiado por fisiologia e os de um CABG guiado por angiografia são diferentes entre si.

Métodos: Pesquisamos nas bases Medline, EMBASE e Cochrane Library. A última data de busca foi junho de 2020, e todos os estudos anteriores foram incluídos. Realizamos uma metanálise de razão de risco agrupado para quatro principais resultados: morte por todas as causas, infarto do miocárdio (IM), revascularização do vaso alvo (TVR) e eventos cardiovasculares adversos maiores (MACE). Valor de $p<0,05$ foi considerado estatisticamente significante. A heterogeneidade foi avaliada com o teste Q de Cochran, e quantificada pelo índice I2.

Resultados: Identificamos cinco estudos incluindo um total de 1.114 pacientes. Uma metanálise agrupada não demonstrou diferenças significativas entre a estratégia da fisiologia e da angiografia para IM (razão de risco [RR] = 0,72; IC95\%, 0,39-1,33; I2 = 0\%; $p=0,65)$, TVR (RR = 1,25; IC95\% = 0,73-2,13; I2 = 0\%; $p=0,52)$, ou MACE (RR = 0,81; IC95\% $=0,62-1,07 ; I 2=0 \% ; p=1)$. A estratégia da fisiologia apresentou 0,63 vezes o risco de morte por todas as causas em comparação à estratégia da angiografia $(R R=0,63 ; I C 95 \%=0,42-0,96 ; I 2=0 \% ; p=0,55)$.

Conclusão: Esta metanálise demonstrou uma redução nas mortes por todas as causas quando usada a estratégia do CABG guiado por fisiologia. Porém, o curto período de acompanhamento, o tamanho da amostra pequeno dos estudos incluídos e a não-discriminação das causas de morte podem justificar essas conclusões. Estudos com períodos mais longos de acompanhamento são necessários para tirar conclusões mais robustas e definitivas.

Palavras-chave: Doença da Artéria Coronariana, Angiografia, Metanálise, Artéria Coronária/fisiologia, Angiografia Coronária, Ponte de Artéria Coronária.

\begin{abstract}
Background: While invasive coronary angiography is considered the gold standard for the diagnosis of coronary artery disease (CAD) involving the epicardial coronary vessels, coronary physiology-guided revascularization represents a contemporary gold-standard practice for the invasive management of patients with intermediate CAD. Nevertheless, the long-term results of assessing the severity of stenosis through physiology compared to the angiogram as the guide to bypass surgery - coronary artery bypass grafting (CABG) are still uncertain. This meta-analysis aims to assess the clinical outcomes of a physiology guided CABG compared to the angiography-guided CABG.
\end{abstract}

Objectives: We sought to determine if outcomes differ between a physiology guided CABG compared to an angiography-guided CABG.

Methods: We searched Medline, EMBASE, and the Cochrane Library. The last date for this search was June 2020, and all of the previous studies were included. We conducted a pooled risk-ratio meta-analysis for four main outcomes: all-cause death, myocardial infarction (MI), target vessel revascularization (TVR) and major adverse cardiovascular events (MACE). P-value $<0.05$ was considered as statistically significant. Heterogeneity was assessed with Cochran's $Q$ test and quantified by the $I^{2}$ index.

Correspondência: José Martins •

Baixo Vouga Hospital Centre - Avenida Ravara, 3814-501, Aveiro - Portugal

E-mail: zeluismartins@gmail.com

Artigo recebido em 11/07/2020, revisado em 11/10/2020, aceito em 04/12/2020

DOI: https://doi.org/10.36660/abc.20200763 
Results: We identified five studies that included a total of 1,114 patients. A pooled meta-analysis showed no significant difference between a physiology guided strategy and an angiography-guided strategy in $\mathrm{MI}$ (risk ratio $[R R]=0.72 ; 95 \% \mathrm{Cl}, 0.39-1.33 ; \mathrm{I} 2=0 \% ; p=0.65$ ), TVR $(R R=1.25 ; 95 \% \mathrm{Cl}=0.73-2.13 ; 12=0 \% ; p=0.52)$, or MACE $(R R=0.81 ; 95 \% \mathrm{Cl}=0.62-1.07 ; 12=0 \% ; p=1)$. The physiology guided strategy has 0.63 times the risk of all-cause death compared to the angiography-guided strategy $(R R=0.63 ; 95 \% \mathrm{Cl}=0.42-0.96 ; 12=0 \% ; p=0.55)$.

Conclusion: This meta-analysis demonstrated a reduction in all-cause death when a physiology guided CABG strategy was used. Nevertheless, the short follow-up period, small sample size of the included studies and the non-discrimination of the causes of death can largely justify these conclusions. Studies with an extended follow-up period of observation are required to draw more robust and definitive conclusions.

Keywords: Coronary Artery Disease; Angiography; Metanalysis; Coronary Artery/physiology; Coronary Angiography; Coronary Artery Bypass.

Full texts in English - http://www.arquivosonline.com.br

\section{Introdução}

A angiografia coronária invasiva é considerada padrão ouro para o diagnóstico da doença arterial coronariana (DAC) envolvendo os vasos coronários epicárdicos. ${ }^{1}$ Porém, a avaliação visual por meio da angiografia coronária tradicional não é capaz de distinguir se uma estenose coronária é hemodinamicamente significativa, principalmente na DAC intermediária; então, há discordâncias constantes entre a gravidade angiográfica e a significância da fisiologia na DAC. ${ }^{2,3}$ As discrepâncias ocorrem porque, diferentemente da angiografia, a fisiologia incorpora os efeitos combinados e inter-relacionados do fluxo coronário e da resistência microvascular. ${ }^{3}$

Há crescentes evidências que apoiam os benefícios clínicos e a custo-efetividade da revascularização coronária percutânea guiada pela fisiologia, seja pelos índices baseados na hiperemia ou pelos índices baseados na diferença de pressão durante um período específico da diástole, em comparação à revascularização percutânea baseada na angiografia coronária. ${ }^{4-7}$ Porém, os resultados de longo prazo da avaliação da gravidade da estenose por meio da fisiologia, em comparação à angiografia como guia para a cirurgia de bypass, ainda são incertos.

Considerando esses resultados, cirurgiões cardíacos estão progressivamente guiando a revascularização da doença de múltiplos vasos (MVD) com base na fisiologia coronária. Porém, ainda não está claro se esta decisão traz melhores resultados clínicos de longo prazo. As recomendações sobre o uso do CABG em comparação à terapia médica ou à intervenção coronária percutânea (ICP) são inteiramente baseadas em estudos que utilizam critérios anatômicos e não-funcionais para guiar a revascularização. ${ }^{8-12}$

Por conta desses dados, muitos autores já avaliaram o potencial benefício clínico da cirurgia de bypass da artéria coronária guiada pela fisiologia, além dos detalhes anatômicos para decisões cirúrgicas. ${ }^{13-20}$ Neste artigo, estendemos o trabalho de Spadaccio et al., ${ }^{20}$ ao agrupar todos os resultados de estudos randomizados e não-randomizados para avaliar o efeito nos resultados clínicos entre o CABG guiado por fisiologia em comparação ao CABG guiado pela angiografia. ${ }^{20}$

\section{Métodos}

\section{Fontes de dados e buscas}

Nós fizemos buscas sistemáticas nas bases Medline, Embase e Cochrane Library, pesquisando artigos publicados relevantes. A última data da busca foi em junho de 2020, e todos os estudos anteriores foram incluídos na busca. Revisões sistemáticas e qualitativas prévias, se disponíveis, foram consideradas para estudos adicionais. Os seguintes termos de busca foram utilizados: "Fisiologia coronária" ou "Fluxo Fracionado de Reserva" ou "FFR" ou "Instant Wave-Free Ratio" ou "iFR" ou "Enxerto de bypass de artéria coronária" ou "CABG". Outros estudos foram buscados por pesquisa manual ou em fontes secundárias, incluindo referências de artigos primários. Não houve restrições de língua na busca.

\section{Seleção dos estudos}

As citações foram primeiramente selecionadas pelo título/resumo por dois revisores independentes (JM e LS), e os manuscritos completos foram avaliados se fossem potencialmente pertinentes. Discordâncias foram resolvidas após chegarem a um consenso. Os artigos identificados foram avaliados pelos mesmos revisores, de acordo com os seguintes critérios de inclusão: artigos com resultados clínicos comparando as duas estratégias para a revascularização no CABG (fisiologia versus angiografia). As disputas relacionadas aos critérios de inclusão foram resolvidas até chegarem a um consenso. Estudos comparando ambas as estratégias que não reportaram os resultados clínicos foram excluídos. Os estudos que não reportaram a estratégia usada em detalhe, embora tivessem a avaliação dos resultados clínicos, também foram excluídos (Figura 1).

\section{Desfechos}

Os desfechos estudados foram: morte por todas as causas, infarto do miocárdio (IM), revascularização do vaso alvo (TVR) e eventos cardiovasculares adversos maiores (MACE) durante o período de acompanhamento.

MACE foi definido como um composto de morte, infarto do miocárdio ou qualquer revascularização de Moscona et al., ${ }^{16}$ Fournier et al; ${ }^{18}$ e como um composto de morte, infarto do miocárdio, derrame ou qualquer revascularização dos estudos FARGO, FUTURE e GRAFFITI durante o período de acompanhamento. ${ }^{13-19}$

\section{Análise estatística}

Para calcular as estimativas do efeito agrupado, utilizamos a variância inversa assumindo um modelo de efeito fixo, e o método de DerSimonian e $\operatorname{Lard}^{21}$ assumindo o modelo do efeito randômico. ${ }^{21} \mathrm{~A}$ homogeneidade entre os estudos foi 


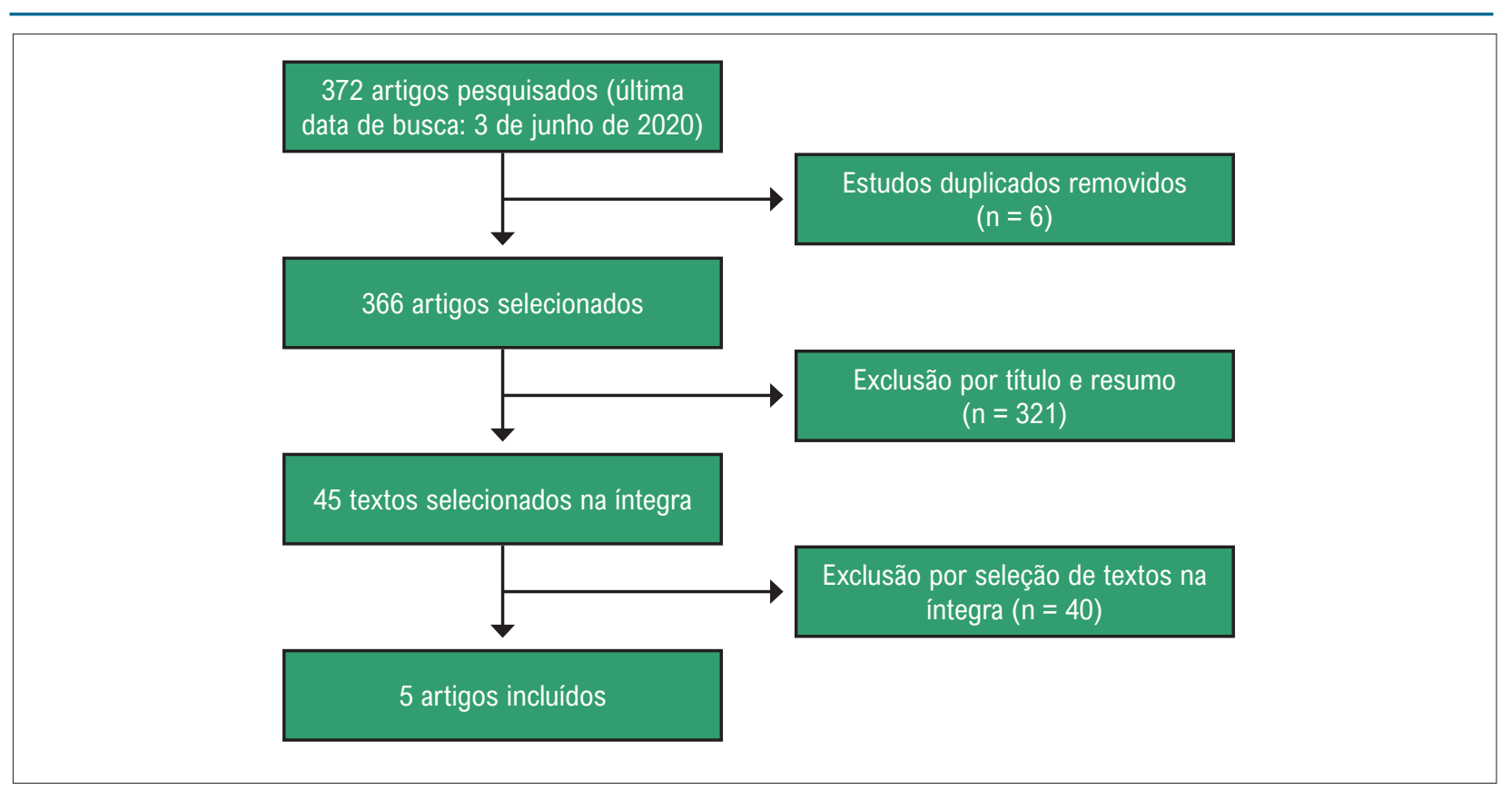

Figura 1 - Fluxo dos estudos incluídos na metanálise.

avaliada com o teste $\mathrm{Q}$ de Cochrane e o índice 12 (os valores de $0,25,0,50$ e 0,75 indicaram níveis baixos, moderados e altos de heterogeneidade, respectivamente). O valor de $p<0,05$ foi considerado estatisticamente significativo. $\mathrm{O}$ viés de publicação foi avaliado utilizando o gráfico em funil. Realizamos uma análise de sensibilidade para mostrar o impacto de cada estudo nos resultados. O MetaXL 2.0 (EpiGear International Pty Ltd, Wilston, Queensland, Australia) foi usado para calcular o tamanho do efeito da diferença do risco agrupado (diferença entre o risco dos grupos com revascularização e com gerenciamento conservador).

\section{Resultados}

\section{Identificação do Estudo}

As buscas nas bases de dados inicialmente encontraram 372 citações. Dessas, seis estudos duplicados e 321 artigos foram excluídos após a revisão do título ou resumo. Depois de uma avaliação aprofundada de acordo com os critérios de seleção, excluímos mais 40 estudos. Um final total de cinco estudos foi incluído na análise. Esses cinco estudos incluíam 1.114 pacientes: 403 no grupo guiado pela fisiologia, e 711 no grupo guiado pela angiografia.

\section{Características dos estudos incluídos}

Dos cinco estudos incluídos, três eram randomizados e dois eram não-randomizados, observacionais e retrospectivos (Tabelas 1 e 2)

\section{Síntese quantitativa dos desfechos}

Morte por todas as causas._A morte por todas as causas foi reportada nos cinco estudos, o que foi considerado para a análise agrupada, para um total de 1.114 pacientes. A estratégia da fisiologia apresentou 0,63 vezes o risco de morte por todas as causas em comparação à estratégia da angiografia $(R R=0,63 ; I C 95 \%=0,42-0,96)$. A Figura 2 descreve a metanálise ponderada para a morte por todas as causas. A análise agrupada demonstrou heterogeneidade insignificante entre os estudos (I2 $=0 \% ; \mathrm{p}=0,55)$. Em uma análise de sensibilidade, ao recalcular os resultados agrupados da análise primária ao excluir cada estudo por vez, no estudo de Fournier et al. esta diferença de risco desaparece. Este efeito também desapareceu quando limitamos a análise a estudos randomizados controlados $(R R=1,09 ; \quad I C 95 \%=0,28-4,3)$. A Figura 3 descreve a metanálise ponderada para a morte por todas as causas quando só os estudos randomizados controlados foram incluídos.

Infarto do miocárdio. Para analisar a ocorrência de IM, quatro estudos que incluíam um total de 1.093 pacientes foram agrupados. Não houve diferença significativa entre as duas estratégias ( $R R=0,72 ;$ IC95\% =0,39-1,33), nem heterogeneidade significativa entre os estudos (I2 = $0 \%$; $p=0,65)$. A exclusão de qualquer estudo único e dos nãorandomizados não alterou o resultado combinado final.

Revascularização do vaso alvo. Para avaliar a TVR, quatro estudos incluindo um total de 1.093 pacientes foram agrupados. Não houve diferença significativa entre as duas estratégias ( $R R=1,25 ; \mathrm{IC} 95 \%=0,73-2,13)$, nem heterogeneidade significativa entre os estudos (I2 = 0\%; $p=0,55)$. A exclusão de qualquer estudo único e dos nãorandomizados não alterou o resultado combinado final.

MACE. Para avaliar os MACE, cinco estudos incluindo 1.114 pacientes foram agrupados. Não houve diferença significativa entre as duas estratégias $(R R=0,81$; 


\begin{tabular}{|c|c|c|c|c|c|c|c|c|}
\hline Autor & Ano & $\begin{array}{c}\mathrm{N} \\
\text { total }\end{array}$ & $\begin{array}{c}\mathrm{N} \text { - } \\
\text { estratégia }\end{array}$ & Acompanhamento & $\begin{array}{l}\text { Desenho do } \\
\text { estudo }\end{array}$ & Vias & $\begin{array}{l}\text { Oclusão do enxerto - } \\
\text { acompanhamento }\end{array}$ & $\begin{array}{c}\text { Principais } \\
\text { conclusões clínicas }\end{array}$ \\
\hline $\begin{array}{l}\text { Moscona } \\
\text { et al. }{ }^{16}\end{array}$ & 2018 & 109 & $\begin{array}{l}\text { Guiado por } \\
\text { FFR/iFR-: } 14 \\
\text { Guiado por } \\
\text { angiografia: } \\
\quad 95\end{array}$ & 18 meses & Retrospectivo & $\begin{array}{c}\text { Via arterial: } \\
\text { 92,9\% } \\
\text { (grupo FFR; } \\
\text { 90,5\% Grupo } \\
\text { angiografia) } \\
\text { EVS: } 85,7 \% \\
\text { (grupo } \\
\text { FFR; } 76,8 \% ; \\
\text { grupo } \\
\text { angiografia) }\end{array}$ & NR & $\begin{array}{c}\text { Uma tendência } \\
\text { de redução da } \\
\text { MACE }(7,1 \% \text { vs. } \\
11,6 \%, P=0,369) \text { e } \\
\text { angina }(0,0 \% \text { vs. } \\
6,3 \%, P=0,429) \text { no } \\
\text { grupo FFR/iFR em } \\
\text { comparação ao } \\
\text { grupo da angiografia. }\end{array}$ \\
\hline $\begin{array}{l}\text { Thuesen } \\
\text { et al. }{ }^{14}\end{array}$ & 2018 & 97 & $\begin{array}{l}\text { Guiado por } \\
\text { FFR: } 49 \\
\text { Guiado por } \\
\text { angiografia: } \\
\quad 48\end{array}$ & Seis meses & $\begin{array}{c}\text { Estudo } \\
\text { controlado } \\
\text { randomizado }\end{array}$ & $\begin{array}{c}\text { Via arterial: } \\
37 \%\end{array}$ & $\begin{array}{l}\text { Falência de enxerto de } \\
\text { todos os enxertos foi } \\
\text { similar em ambos os } \\
\text { grupos ( } 16 \% \text { vs. } 12 \% \text {; } \\
\qquad p=0,97)\end{array}$ & $\begin{array}{c}\text { Taxas de morte, IM, } \\
\text { e derrame foram } \\
\text { similares nos grupos } \\
\text { do estudo. } \\
\text { A mortalidade por } \\
\text { todas as causas } \\
\text { após seis meses } \\
\text { foi de } 0 \% \text { no grupo } \\
\text { FFR, e } 4,1 \% \text { no } \\
\text { grupo angiografia; } \\
\text { um paciente morreu } \\
\text { por conta de } \\
\text { embolia pulmonar, } \\
\text { e um morreu de } \\
\text { mediastinite. }\end{array}$ \\
\hline $\begin{array}{l}\text { Rioufol et } \\
\text { al. }{ }^{17}\end{array}$ & 2018 & 109 & $\begin{array}{l}\text { Guiado por } \\
\text { FFR: } 55 \\
\text { Guiado por } \\
\text { angiografia: } \\
\quad 54\end{array}$ & Um ano & $\begin{array}{l}\text { Estudo } \\
\text { randomizado } \\
\text { controlado }\end{array}$ & NR & NR & $\begin{array}{l}\text { FFR em pacientes } \\
\text { com doença de } \\
\text { múltiplos vasos não } \\
\text { demonstrou melhora } \\
\text { no desfecho do } \\
\text { composto primário } \\
\text { de mortalidade por } \\
\text { todas as causas, } \\
\text { IM, revascularização } \\
\text { repetida ou derrame } \\
\text { por um ano (14,6\% } \\
\text { vs } 14,4 \% ; \text { HR } 0,97 ; \\
\text { IC95\% 0,69-1,36) } \\
\text { Risco de morte foi } \\
\text { significativamente } \\
\text { maior no grupo FFR } \\
\text { (3,7\% vs } 1,5 \% ; P= \\
0,036)\end{array}$ \\
\hline $\begin{array}{l}\text { Fournier } \\
\text { et al. } .^{15}\end{array}$ & 2019 & 627 & $\begin{array}{l}\text { Guiado por } \\
\text { FFR: } 198 \\
\text { Guiado por } \\
\text { angiografia: } \\
429\end{array}$ & Seis anos & Retrospectivo & $\begin{array}{c}\text { Via arterial: } \\
64 \% \\
\text { EVS: } 36 \%\end{array}$ & $\begin{array}{l}\text { A taxa de oclusão foi } \\
\text { significativamente } \\
\text { mais baixa no grupo } \\
\text { FFR em comparação } \\
\text { ao da angiografia }\end{array}$ & $\begin{array}{c}\text { CABG guiado por } \\
\text { FFR está associado à } \\
\text { redução significative } \\
\text { na taxa de morte } \\
\text { geral ou infarto do } \\
\text { miocárdio (HR 0,59 } \\
\text { [IC95\%, 0,38-0,93]; } \\
P=0,020)\end{array}$ \\
\hline $\begin{array}{l}\text { Toth et } \\
\text { al. }^{19}\end{array}$ & 2019 & 172 & $\begin{array}{l}\text { Guiado por } \\
\text { FFR: } 88 \\
\text { Guiado por } \\
\text { angiografia: } \\
84\end{array}$ & Um ano & $\begin{array}{l}\text { Estudo } \\
\text { randomizado } \\
\text { controlado }\end{array}$ & $\begin{array}{l}\text { NR } \\
\text { Taxa da Via } \\
\text { arterial-para- } \\
\text { EVS: } 1: 1\end{array}$ & $\begin{array}{l}\text { Não houve diferença } \\
\text { na oclusão geral dos } \\
\text { enxertos }(80 \% \text { vs } \\
81 \%) p=0,885)\end{array}$ & $\begin{array}{l}\text { Não houve diferença } \\
\text { no composto } \\
\text { morte, infarto do } \\
\text { miocárdio, vaso algo, } \\
\text { revascularização e } \\
\text { derrame (HR 1,275; } \\
\text { IC95\%: } 0,391 \text { a } \\
4,160, p=0,674)\end{array}$ \\
\hline
\end{tabular}

FFR: Fluxo fracionado de reserva; iFR: proporção instantânea de ondas livres; N: pacientes incluídos no estudo; IM: infarto do miocárdio; TVR: revascularização do vaso alvo; NR: não reportado; EVS: enxerto de veia safena; CABG: enxerto de bypass de artéria coronária. Valor de $p<0,05$ foi considerado significativo em todos os estudos incluidos. 


\begin{tabular}{|c|c|c|c|c|c|c|c|}
\hline Autor & Ano & $\mathrm{N}$ total & $\mathrm{N}$ - estratégia & Morte & IM & TVR & MACE \\
\hline \multirow{2}{*}{$\begin{array}{l}\text { Moscona et } \\
\text { al. }^{16}\end{array}$} & \multirow{2}{*}{2018} & \multirow{2}{*}{109} & Guiado por FFR/iFR: 14 & FFR/iFR: 1 & FFR/iFR: 0 & FFR/iFR: 0 & FFR/iFR: 1 \\
\hline & & & Guiado por angiografia: 95 & Angiografia: 5 & Angiografia: 2 & Angiografia: 4 & Angiografia: 11 \\
\hline \multirow{2}{*}{$\begin{array}{l}\text { Thuesen et } \\
\text { al. }{ }^{14}\end{array}$} & \multirow{2}{*}{2018} & \multirow[t]{2}{*}{97} & Guiado por FFR: 49 & FFR: 0 & FFR: 1 & FFR: 2 & FFR: 6 \\
\hline & & & Guiado por angiografia: 48 & Angiografia: 2 & Angiografia: 0 & Angiografia: 0 & Angiografia: 6 \\
\hline \multirow{2}{*}{$\begin{array}{l}\text { Rioufol et } \\
\text { al. } .^{17}\end{array}$} & \multirow{2}{*}{2018} & \multirow{2}{*}{109} & Guiado por FFR: 55 & FFR: 1 & \multirow{2}{*}{ NR } & \multirow{2}{*}{ NR } & \multirow{2}{*}{$\begin{array}{c}\text { HR } 0 ., 45(0,108- \\
6,612)\end{array}$} \\
\hline & & & Guiado por angiografia: 54 & Angiografia: 0 & & & \\
\hline \multirow{2}{*}{$\begin{array}{l}\text { Fournier et } \\
\text { al. }{ }^{15}\end{array}$} & \multirow{2}{*}{2019} & \multirow{2}{*}{627} & $\begin{array}{l}\text { Guiado por FFR: } 198 \\
\text { Guiado por angiografia: }\end{array}$ & FFR: 21 & FFR: 11 & FFR: 17 & FFR: 42 \\
\hline & & & 429 & Angiografia: 79 & Angiografia: 34 & Angiografia: 27 & Angiografia: 113 \\
\hline \multirow{2}{*}{ Toth et al. ${ }^{19}$} & \multirow{2}{*}{2019} & \multirow{2}{*}{172} & \multirow{2}{*}{$\begin{array}{c}\text { Guiado por FFR: } 88 \\
\text { Guiado por angiografia: } 84\end{array}$} & FFR: 3 & FFR: 0 & FFR: 2 & FFR: 5 \\
\hline & & & & Angiografia: 2 & Angiografia: 2 & Angiografia: 4 & Angiografia: 6 \\
\hline
\end{tabular}

IM: infarto do miocárdio; TVR: revascularização do vaso alvo

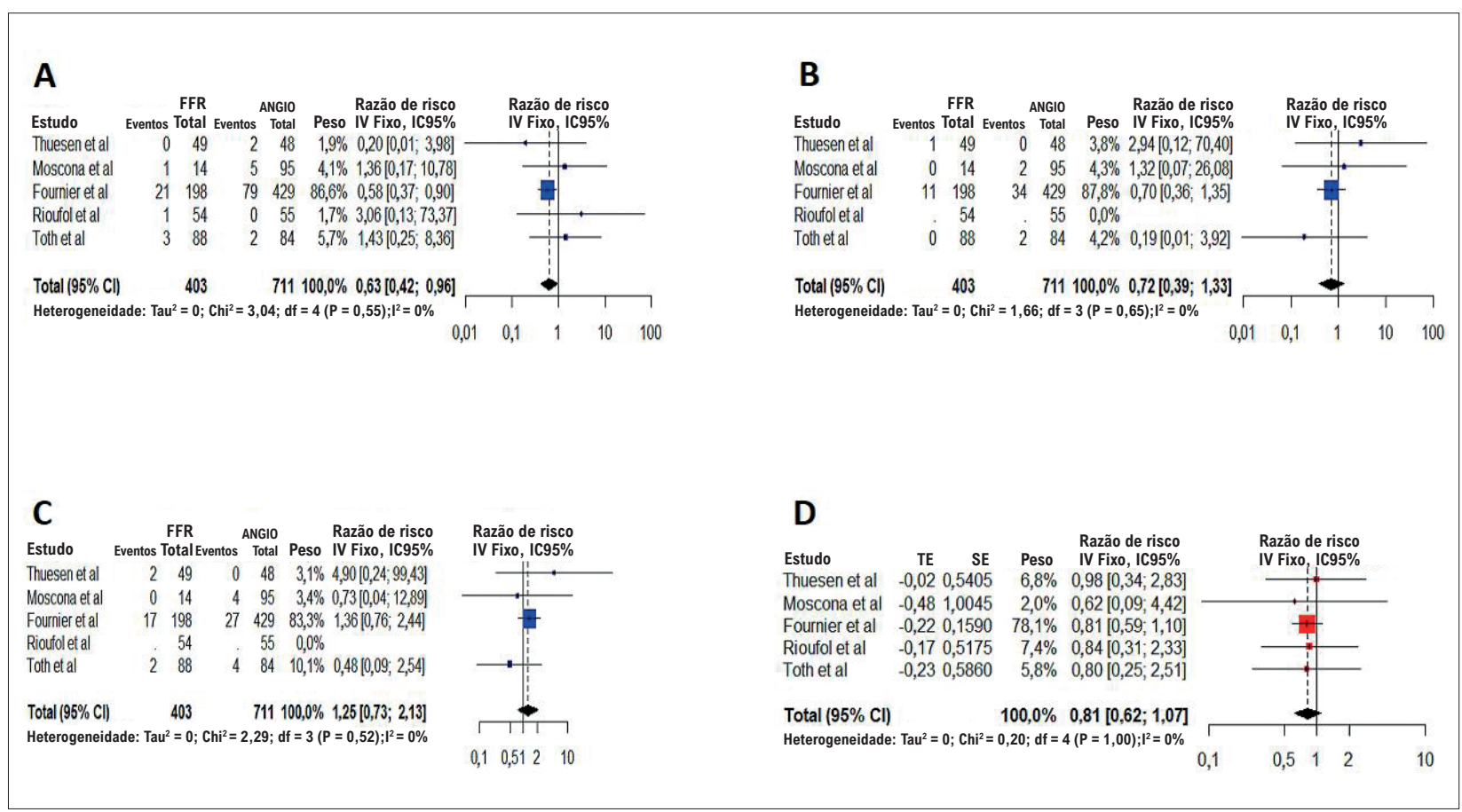

Figura 2 - Gráfico em Floresta da razão de risco agrupada pelos desfechos: (A) morte por todas as causas; (B) IM; (C) TVR; (D) MACE. O tamanho dos marcadores de dados indica o peso do estudo. IC: intervalo de confiança; IM: infarto do miocárdio; TVR: revascularização do vaso alvo.

IC95\% = 0,62-1,07), nem heterogeneidade significativa entre os estudos ( $I 2=0 \% ; p=1)$. A exclusão de qualquer estudo único e dos não-randomizados não alterou o resultado combinado final.

\section{Viés do estudo}

A inspeção visual do gráfico de funil para os desfechos não revelou nenhuma assimetria entre os estudos (Figura 4). Além disso, o teste de correlação de Begg não foi estatisticamente significante.

\section{Discussão}

A sobrevivência tem uma correlação significativamente negativa com a carga obstrutiva da DAC angiográfica. O escore SYNTAX estratifica a complexidade angiográfica da doença arterial coronariana e estabelece o prognóstico de pacientes com MVD, sendo uma ferramenta importante para decidir sobre a melhor estratégia de revascularização. ${ }^{22,23}$

Há discordâncias entre a gravidade da significância da angiografia e da fisiologia da DAC, então, o escore SYNTAX, que é obtido ao considerar somente as lesões provocadas por 
A

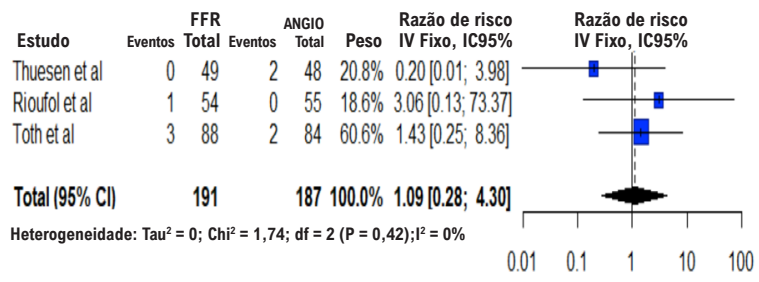

C

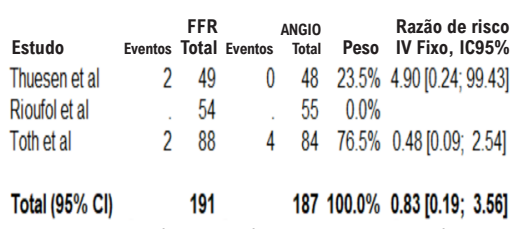

Heterogeneidade: $\mathrm{Tau}^{2}=1685 ; \mathrm{Chi}^{2}=1,76 ; \mathrm{df}=1(\mathrm{P}=0,18) ; \mathrm{l}^{2}=43 \%$

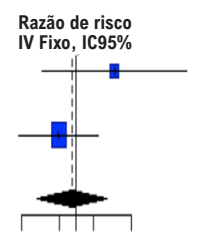

0.10 .51210
B

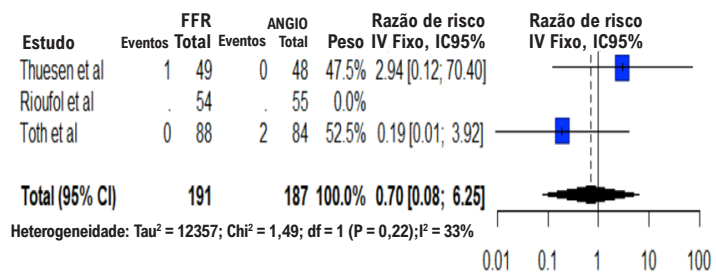

D

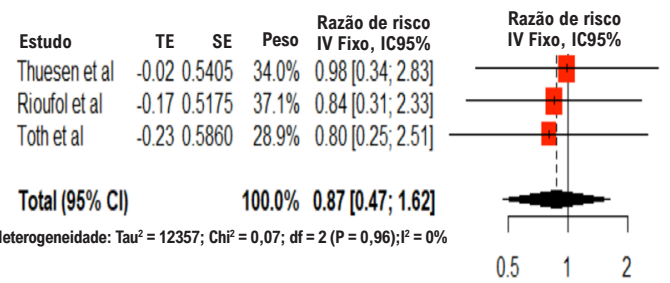

Figura 3 - Gráfico em Floresta da razão de risco agrupada para os desfechos quando somente os estudos randomizados controlados foram incluídos: (A) morte por todas as causas; (B) IM; (C) TVR; (D) MACE. 0 tamanho dos marcadores de dados indica o peso do estudo. IC: intervalo de confiança; IM: infarto do miocárdio; TVR: revascularização do vaso alvo.

A

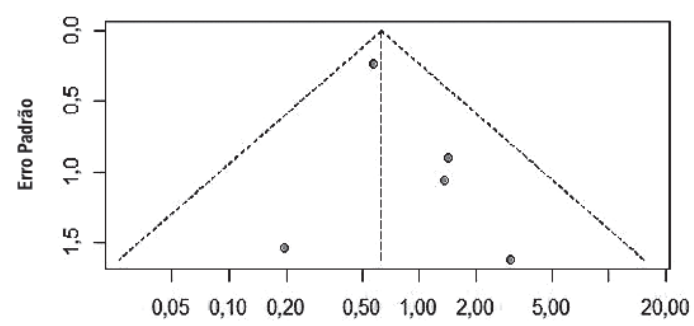

C

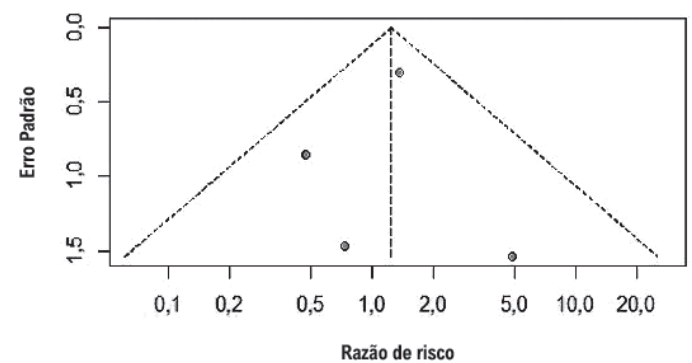

B

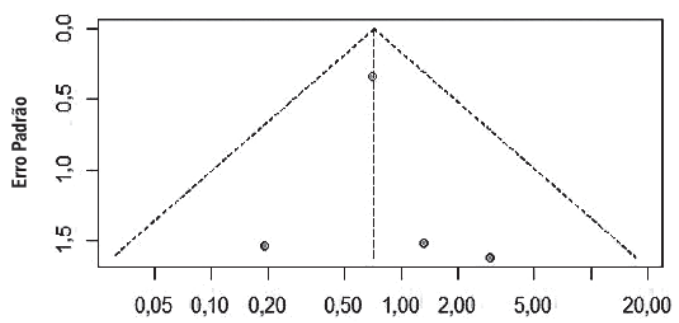

D

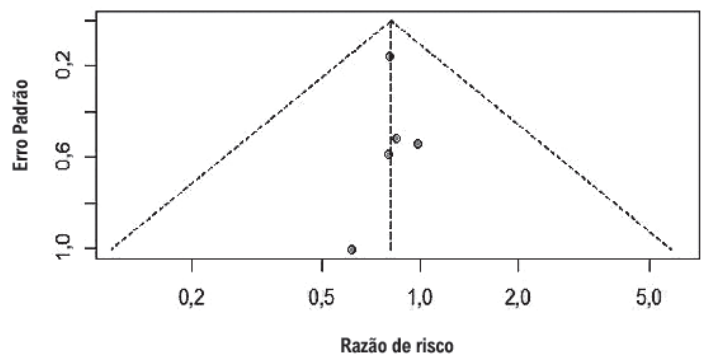

Figura 4 - Viés de publicação para: (A) morte por todas as causas; (B) IM; (C) TVR; (D) MACE. Círculos representam estudos individuais da metanálise, e a linha vertical representa a estimativa agrupada da Razão de Risco para morte por todas as causas, IM, TVR e MACE. IM: infarto do miocárdio; TVR: revascularização do vaso alvo. 
isquemia, pode superar esta limitação. Em comparação com o clássico escore SYNTAX, o escore SYNTAX funcional tem melhor reprodutibilidade e valor prognóstico, reclassificando até $32 \%$ dos candidatos ao CABG e gerando implicações em termos de direcionamento terapêutico. ${ }^{22-24}$

Se os impactos favoráveis da fisiologia coronariana nos desfechos da ICP podem ou não ser traduzidos em prática cirúrgica tornou-se o tema de nossa investigação.

Nossa metanálise mostrou uma redução de 37\% das mortes por todas as causas no grupo guiado pela fisiologia, com redução não estatisticamente significativa em IM e MACE; esses desfechos não estiveram associados ao aumento do TVR. Esses resultados devem ser interpretados considerando os limites intrínsecos a cada um dos estudos, incluindo viés de seleção, já que esta redução desaparece quando somente os estudos randomizados controlados são agrupados para análise.

Ao avaliar os desfechos clínicos na revascularização, há importantes considerações a serem feitas. A primeira é relacionada aos desfechos (peri) procedimento. O tipo de IM deve estar claramente estabelecido ao comparar as estratégias, já que hoje é universalmente aceito que o prognóstico do IM espontâneo não é similar ao IM periprocedimento ou o IM tipo 2 . $^{25,26}$

A história natural da doença é outro ponto importante a ser considerado como novo paradigma, com o foco na doença em si (aterosclerose) e não no sintoma (isquemia). ${ }^{25,27}$ A composição da placa, avaliada por algumas características da imagem, aparentemente é o principal determinante do prognóstico, até mais do que o nível da estenose coronária ou sua localização. ${ }^{28-30}$ Isso pode explicar o melhor prognóstico associado à completa revascularização no contexto da síndrome coronária aguda, na qual as placas em lesões não culpadas aparentemente possuem características instáveis, contrastando com os achados na doença coronariana estável. ${ }^{31}$

A terceira consideração importante é o tipo de revascularização. Os benefícios bem definidos do CABG em comparação à ICP guiada pela angiografia, como reportado nos estudos ASCERT, SYNTAX, FREEDOM e BEST, usam critérios anatômicos e não-funcionais para guiar a revascularização e vêm antes da tecnologia de stent farmacológico da nova geração..$^{8-12}$

Uma diferença considerável entre o CABG e a ICP depende dos efeitos protetivos da progressão da doença aterosclerótica. Sabe-se que a maioria das estenoses relacionadas ao IM está localizada no terço proximal da árvore coronária. Também sabe-se que a maioria dos IMs advém de placas nãosignificantes. Enxertos cirúrgicos de bypass normalmente são implantados distalmente na circulação coronária, promovendo "um efeito de colateralização" na revascularização, e parece concebível que o benefício prognóstico do CABG possa ser explicado pela proteção contra eventos coronários, independentemente da gravidade da estenose do vaso enxertado. O conceito de revascularização baseado na fisiologia, e não no tipo de placa, elimina o efeito protetor do bypass cirúrgico. ${ }^{28-30,32}$

O conceito da completa revascularização surgiu de estudos prévios sobre o CABG, enquanto algumas publicações demonstravam que pacientes que estavam completamente revascularizados tinham um benefício em termos de mortalidade em relação àqueles que estavam revascularizados de forma incompleta, o que estabeleceu um padrão para o campo do CABG. ${ }^{33-35}$

A revascularização baseada na fisiologia também traz o conceito da revascularização completamente anatômica versus funcional. Se, por um lado, o uso da fisiologia coronária reduz o número de vasos enxertados, ou até de cirurgias, sem a circulação extracorpórea, por outro aumenta a taxa de revascularização incompleta anatomicamente definida. ${ }^{19}$

O uso da revascularização guiada pela fisiologia demonstrou reduzir a taxa de MACE nos pacientes com MVD, com ponto de corte de FFR de 0,78, mostrando associação significativa entre a medida de FFR pré-operatório do vaso alvo e a funcionalidade da anastomose após seis meses. Essas conclusões também são apoiadas por Botman et al., ${ }^{36}$ para quem as lesões com FFR $>0,75$ estão associadas ao aumento significativo do risco de oclusão do enxerto ( $p$ $<0,0001){ }^{18,19,36,37}$

Considerando as diferenças descritas na suscetibilidade à competitividade de fluxo, parece provável que o tipo de conduta usada nos estudos FARGO e GRAFFITI (nos quais uma grande proporção dos enxertos eram enxerto de veia safena - EVS) versus o estudo IMPAG (no qual somente enxertos arteriais eram utilizados) possa explicar os resultados contraditórios. ${ }^{14,19,38}$

O estudo FAME 3 vai comparar, de forma multicêntrica e randomizada, a ICP guiada por FFR com stents farmacológicos contemporâneos com o CABG em pacientes com a doença de 3 vasos. Porém, não vai responder sobre a comparação entre o CABG guiado por fisiologia e o CABG guiado pela angiografia. ${ }^{39}$

Finalmente, o papel do acompanhamento neste contexto também deve ser considerado. Quanto mais grave a estenose coronária, maior o risco de $\mathrm{IM}$, mas são as placas nãosignificativas as responsáveis pela maioria dos IMs. Também sabemos que a principal causa de morte nesses pacientes com DAC é relacionada ao coração, e que o IM é uma causa de morte cardíaca; então, as terapias para reduzir o IM ou a morte cardiovascular vão, consequentemente, reduzir a mortalidade. $^{32}$

Podemos considerar o estudo STICH como exemplo, comparando o tratamento com terapia clínica somado ao CABG e somente a terapia clínica em pacientes com DAC e insuficiência cardíaca, com fração de ejeção reduzida. Após cinco anos de acompanhamento, a análise da intenção de tratamento não demonstrou diferenças significativas entre as duas estratégias com relação ao desfecho primário de morte por todas as causas. Porém, após o período de acompanhamento ter sido estendido para dez anos, uma redução significativa na mortalidade foi encontrada para o CABG somado à terapia clínica, em comparação à terapia clínica somente $(H R, 0,84$; IC95\%: 0,73-0,97; $p=0,02)$. Outro exemplo é o estudo FAME 2, no qual os dados publicados após cinco anos mostram uma forte tendência a baixas taxas de infarto do miocárdio no grupo ICP (HR 0,66; IC95\% 0,43-1,00; p = 0,049), uma diferença que só foi significativa para o IM espontâneo (HR 0,62; IC95\%; $p=0,04)$, e não para o IM periprocedimento. 
Recentemente, o estudo ISCHEMIA mostrou que, em um acompanhamento prévio, o desfecho composto primário (morte cardiovascular, IM ou hospitalização devido à angina instável ou insuficiência cardíaca) foi mais frequente no grupo com a estratégia invasiva do que no grupo da estratégia conservadora (5,3\% vs. 3,4\% após seis meses), devido aos IMs relacionados ao procedimento. Porém, em um acompanhamento posterior, depois de aproximadamente dois anos, as curvas dos eventos se cruzaram e, após cinco anos, a incidência do desfecho primário foi um pouco maior no grupo da estratégia conservadora (18,2\% e 16,4\%). Então, parece que para se ter um impacto nos desfechos duros, como morte por todas as causas, temos que estender a duração do período de observação. ${ }^{25,40,41}$

Em nosso estudo, a redução da morte por todas as causas em um contexto de redução não significativa do IM e MACE deve ser interpretada com cautela, já que esta situação pode estar relacionada a mortes causadas por motivos relacionados ao coração. Seria interessante não só avaliar se essas mortes têm relação com o coração, mas também estender o acompanhamento dos estudos para observar se as curvas dos desfechos duros divergem em períodos mais longos, possibilitando chegar a conclusões definitivas. É importante observar que somente o estudo de Fournier et al., ${ }^{18}$ tem um período de acompanhamento mais longo do que cinco anos, o que pode explicar os resultados, já que a redução no desfecho composto morte por todas as causas e IM, apoiando a estratégia da fisiologia, só foi encontrada quando o período de acompanhamento foi estendido. ${ }^{15}$

\section{Limitações}

As conclusões tiradas desta metanálise estão sujeitas às limitações e diferenças dos estudos originais incluídos na análise. A limitação desta metanálise é a presença de estudos com amostras menores e abrangentes, e resultados de sobrevivência de longo prazo. Outra limitação é representada por um viés de seleção intrínseco. Diversas decisões sobre revascularização na estratégia guiada pela fisiologia foram desviadas da indicação funcional nos estudos incluídos, e os autores justificaram que isso esteve relacionado a causas técnicas e, em alguns casos, à relutância para adiar a revascularização. Como mencionado, como a causa de morte não era conhecida, isso inclui mortes não relacionadas ao coração, ou seja, não ligadas à escolha da estratégia da revascularização. Outra limitação foi que com a inclusão de dois estudos retrospectivos e observacionais, alguns pacientes incluídos neste registro podem ter sido tratados com ICP guiada pela fisiologia, e não pelo CABG.

\section{Conclusão}

Esta metanálise demonstra uma redução na morte por todas as causas quando a estratégia do CABG guiado pela fisiologia foi utilizada. Porém, o curto período de acompanhamento, a amostra pequena dos estudos incluídos e a não-discriminação de causas de morte podem justificar essas conclusões. Estudos com períodos mais longos de observação são necessários para se chegar a conclusões mais robustas e definitivas.

\section{Contribuição dos autores}

Concepção e desenho da pesquisa: Martins J, Santos L; Obtenção de dados e Redação do manuscrito: Martins J; Análise e interpretação dos dados e Análise estatística: Martins J, Afreixo V; Revisão crítica do manuscrito quanto ao conteúdo intelectual importante: Martins J, Santos L, Fernandes L, Briosa A.

\section{Potencial conflito de interesse}

Não há conflito com o presente artigo

\section{Fontes de financiamento}

O presente estudo não teve fontes de financiamento externas.

\section{Vinculação acadêmica}

Não há vinculação deste estudo a programas de pósgraduação.

\section{Aprovação ética e consentimento informado}

Este artigo não contém estudos com humanos ou animais realizados por nenhum dos autores.

\section{Referências}

1 Knuuti J, Wijns W, Saraste A, Capodanno D, Barbato E, Funck-Brentano C, et al. 2019 ESC Guidelines for the diagnosis and management of chronic coronary syndromes: The Task Force for the diagnosis and management of chronic coronary syndromes of the European Society of Cardiology (ESC). Eur Heart J. 2020;41(3):407-77.

2. Masdjedi K, Daemen J. Angiography-Based FFR: Reevaluating the Role of the Pressure Wire. Card Interv Today. 2019;13(3):28-33.

3. Morris PD, Curzen N, Gunn JP. Angiography-derived fractional flow reserve: more or less physiology?.J Am Heart Assoc. 2020;9(6):e015586.

4. Neumann FJ, Sousa-Uva M, Ahlsson A, Alfonso F, Banning AP, Benedetto $U$, et al. 2018 ESC/EACTS guidelines on myocardial revascularization. Eur Heart J. 2019;40(2):87-165.

5. De Bruyne B, Pijls NH, Kalesan B, Barbato E, Tonino PAL, Piroth Z, et al Fractional flow reserve-guided $\mathrm{PCl}$ versus medical therapy in stable coronary disease. N Engl J Med. 2012;367:991-1001.

6. Pijls NH, Schaardenburgh P, Manoharan G, Boersma E, Bech JW, van't Veer $\mathrm{M}$, et al. Percutaneous coronary intervention of functionally nonsignificant stenosis: 5-year follow-up of the DEFER study. J Am Coll Cardiol. 2007;49(21):2105-11.

7. Tonino PA, De Bruyne B, Pijls NH, Siebert U, Ikeno F, van't Veer M, et al. Fractional flow reserve versus angiography for guiding percutaneous coronary intervention. N Engl J Med. 2009;360(3):213-24.

8. Weintraub WS, Grau-Sepulveda MV, Weiss JM, O'Brien SM, Peterson ED, Kolm P, et al. Comparative effectiveness of revascularization strategies. $\mathrm{N}$ Engl J Med. 2012;366(16):1467-76. 
9. Serruys PW, Morice MC, Kappetein AP, Colombo A, Holmes DR, Mack MJ, et al. Percutaneous coronary intervention versus coronary-artery bypass grafting for severe coronary artery disease. N Engl J Med. 2009;360(10):961-72.

10. Mohr FW, Morice MC, Kappetein AP, Feldman TE, Ståhle E, Colombo A, et al. Coronary artery bypass graft surgery versus percutaneous coronary intervention in patients with three-vessel disease and left main coronary disease: 5-year follow-up of the randomised, clinical SYNTAX trial. Lancet. 2013;381(9867):629-38.

11. Farkouh ME, Domanski M, Sleeper LA, Siami FS, Dangas G, Mack M, et al. Strategies for multivessel revascularization in patients with diabetes. N Engl J Med. 2012;367(25):2375-84

12. Park SJ, Ahn JM, Kim YH, Park DW, Yun SC, Lee JY, et al. Trial of everolimuseluting stents or bypass surgery for coronary disease. N Engl J Med. 2015;372(13):1204-12.

13. Toth G, De Bruyne B, Casselman F, De Vroey F, Pyxaras S, Serafino L, et al. Fractional flow reserve-guided versus angiography-guided coronary artery bypass graft surgery. Circulation. 2013;128(13):1405-11.

14. Thuesen AL, Riber LP, Veien KT, Christiansen EH, Jensen SE, Modrau I, et al. Fractional flow reserve versus angiographically-guided coronary artery bypass grafting. J Am Coll Cardiol. 2018;72(22):2732-43.

15. Fournier S, Toth GG, De Bruyne B, Johnson NP, Ciccarelli G, Xaplanteris P, et al. Six-year follow-up of fractional flow reserve-guided versus angiographyguided coronary artery bypass graft surgery. Circ Cardiovasc Interv. 2018;11(6):e006368.

16. Moscona JC, Stencel JD, Milligan G, Salmon C, Maini R, Katigbak P, et al. Physiologic assessment of moderate coronary lesions: a step towards complete revascularization in coronary artery bypass grafting. Ann Transl Med. 2018;6(15):300

17. Rioufol G. FUTURE trial: Treatment strategy in multivessel coronary disease patients based on fractional flow reserve. Presented at: European Society of Cardiology (ESC) 2018. August 25, 2018. Munich, Germany.

18. Fournier S, Toth GG, Colaiori I, De Bruyne B, Barbato E. Long-term patency of coronary artery bypass grafts after fractional flow reserve-guided implantation. Circ Cardiovasc Interv. 2019;12(5):e007712.

19. Toth GG, De Bruyne B, Kala P, Ribichini FL, Casselman F, Ramos R, et al. Graft patency after FFR-guided versus angiography-guided coronary artery bypass grafting. The GRAFFITI trial. Eurolntervention. 2019;15(11):e999-1005.

20. Spadaccio C, Glineur D, Barbato E, Di Franco A, Oldroyd KG, BiondiZoccai G, et al. Fractional Flow Reserve-Based Coronary Artery Bypass Surgery: Current Evidence and Future Directions. JACC Cardiovasc Interv. 2020;13(9):1086-96.

21. DerSimonian R, Laird N. Meta-analysis in clinical trials. Control Clin Trials. 1986;7(3):177-88

22. Serruys PW, Morice MC, Kappetein AP, Colombo A, Holmes DR, Mack MJ, et al. Percutaneous coronary intervention versus coronary-artery bypass grafting for severe coronary artery disease. N Engl J Med. 2009;360(10):961-72.

23. Thuijs DJFM, Kappetein AP, Serruys PW, Mohr FW, Morice MC, Mack MJ, et al. Percutaneous coronary intervention versus coronary artery bypass grafting in patients with three-vessel or left main coronary artery disease: 10-year follow-up of the multicentre randomised controlled SYNTAX trial. Lancet. 2019;394(10206):1325-34.

24. Serruys PW, Kogame N, Katagiri Y, Modolo R, Buszman PE, Íñiguez-Romo $A$, et al. Clinical outcomes of state-of-the-art percutaneous coronary revascularisation in patients with three-vessel disease: two-year follow-up of the SYNTAX II study. Eurolntervention. 2019;15(3):e244-52.

25. Maron DJ, Hochman JS, Reynolds HR, Bangalore S, O'Brien SM, Boden WE, et al. Initial Invasive or Conservative Strategy for Stable Coronary Disease. N Engl J Med. 2020;382:1395-407.
26. Ben-Yehuda O, Chen S, Redfors B, McAndrew T, Crowley A, Kosmidou J, et al. I Impact of large periprocedural myocardial infarction on mortality after percutaneous coronary intervention and coronary artery bypass grafting for left main disease: an analysis from the EXCEL trial. Eur HeartJ. 2019;40(24):1930-41.

27. Lardinois CK. Atherosclerosis - Know your risk -is time for a paradigm shift? US Endocrinol. 2014;10(2):105-10.

28. Jeon C, Candia SC, Wang JC, Holper EM, Ammerer M, Kuntz RE, et al. Relative spatial distributions of coronary artery bypass graft insertion and acute thrombosis: a model for protection from acute myocardial infarction. Am Heart J. 2010;160(1):195-201.

29. Wang JC, Normand SL, Mauri L, Kuntz RE. Coronary artery spatial distribution of acute myocardial infarction occlusions. Circulation. 2004;110(3):278-84.

30. Mushtaq S, Goncalves P, Garcia-Garcia HM, Pontone G, Bartorelli A, Bertella $\mathrm{E}$, et al. Long-term prognostic effect of coronary atherosclerotic burden: validation of the computed tomography-Leaman score. Circ Cardiovasc Imaging. 2015;8(2):e002332.

31. Al-Abdouh A, Barbarawi M, Bizanti A, Abudaya I, Upadhrasta S, Elias H et al. Complete revascularization in patients with STEMI and multi-vesse disease: A meta-analysis of randomized controlled trials. Cardiovasc Revasc Med. 2020;21(5):684-91.

32. Doenst $T$, Haverich $A$, Serruys $P$, Bonow RO, Kappetein P, Falk V, et al. PCl and $C A B G$ for treating stable coronary artery disease: JACC Review Topic of the Week. J Am Coll Cardiol. 2019;73(8):964-76.

33. Buda AJ, Macdonald IL, Anderson MJ, Strauss HD, David TE, Berman ND. Long-term results following coronary bypass operation. Importance of preoperative actors and complete revascularization. J Thorac Cardiovasc Surg. 1981;82(3):383-90

34. Bell MR, Gersh BJ, Schaff HV, Holmes Jr DR, Fisher LD, Alderman EL, et al. Effect of completeness of revascularization on long-term outcome of patients with three-vessel disease undergoing coronary artery bypass surgery. A report from the Coronary Artery Surgery Study (CASS) Registry. Circulation. $1992 ; 86(2): 446-57$

35. Ong AT, Serruys PW. Complete revascularization: coronary artery bypas graft surgery versus percutaneous coronary intervention. Circulation. 2006;114(3):249-55.

36. Botman K, Pijls NH, Bech JW, Aarnoudse W, Peels K, Straten BV, et al Percutaneous coronary intervention or bypass surgery in multivessel disease? A tailored approach based on coronary pressure measurement. Catheter Cardiovasc Interv. 2004;63(2):184-91.

37. Kern MJ, Seto AH. High FFR strongly predicts arterial graft dysfunction: pure benefit in a pure population? Eur Heart J. 2019;40(29):2429-31.

38. Glineur D, Grau JB, Etienne PY, Benedetto U, Fortier JH, Papadatos S, et al. Impact of preoperative fractional flow reserve on arterial bypass graft anastomotic function: the IMPAG trial. Eur Heart J. 2019;40(29):2421-28.

39. Zimmermann FM, De Bruyne B, Pijls NH, Desai M, Oldroyd KG, Park SJ, et al. Rationale and design of the Fractional Flow Reserve versus Angiography for Multivessel Evaluation (FAME) 3 Trial: a comparison of fractional flow reserve-guided percut aneous coronary intervention and coronary artery bypass graft surgery in patients with multivessel coronary artery disease. Am Heart J. 2015;170(4):619-26.e2.

40. Petrie MC, Jhund PS, She L, Adlbrecht C, Doenst T, Panza JA, et al. Ten-Year Outcomes After Coronary Artery Bypass Grafting According to Age in Patients With Heart Failure and Left Ventricular Systolic Dysfunction: An Analysis of the Extended Follow-Up of the STICH Trial (Surgical Treatment for Ischemic Heart Failure). Circulation. 2016;134(18):1314-24.

41. Xaplanteris P, Fournier S, Pijls NHJ, Fearon WF, Barbato E, Tonino PAL, et al. Five- year outcomes with $\mathrm{PCl}$ guided by fractional flow reserve. $\mathrm{N} \mathrm{Engl} \mathrm{J}$ Med. 2018;379(3):250-9. 